MANCHESTER

1824

넌

Economics

旅

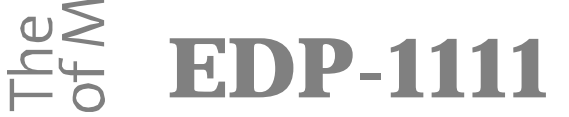

\title{
Accounting for Optimism and Pessimism in Expected Utility
}

Craig Webb

Horst Zank

February 2011

Economics

School of Social Sciences

The University of Manchester

Manchester M13 9PL 


\title{
Accounting for Optimism and Pessimism in Expected Utility
}

\author{
by Craig S. Webb and Horst Zank*
}

21 February 2011

\begin{abstract}
We provide a preference foundation for decision under risk resulting in a model where probability weighting is linear as long as the corresponding probabilities are not extreme (i.e., 0 or 1 ). This way, most of the elegance and mathematical tractability of expected utility is maintained and also much of its normative foundation. Yet, the new model can accommodate the extreme sensitivity towards changes from 0 to almost impossible and from almost certain to 1 that has widely been documented in the experimental literature. The model can be viewed as "expected utility with the best and worst in mind" as suggested by Chateauneuf, Eichberger and Grant (J. Econ. Theory 2007) or, following our preference foundation, interpreted as "expected utility with consistent optimism and pessimism."
\end{abstract}

Keywords: Expected utility, optimism and pessimism, probability weighting function, preference foundation, prospect theory, rank-dependent utility.

Journal of Economic Literature Classification Numbers: D81.

\footnotetext{
${ }^{*}$ Correspondence: Horst Zank, Economics, School of Social Sciences, The University of Manchester, Oxford Road, Manchester M13 9PL, United Kingdom; Telephone: ++44 161275 4872, Fax: ++44 1612754812 , E-mail: horst.zank@manchester.ac.uk
}

We thank Peter Wakker, Aurélien Baillon and Kirsten Rohde for useful comments and helpful discussions. 


\section{Introduction}

It is well-known in the literature on decision under uncertainty and risk that people view certain and impossible events markedly different from likely or possible events, respectively. In particular, very small deviations from certain or from impossible events have significantly more impact on choice behavior than similar deviations from other likely events. People are optimistic about unlikely good news events but pessimistic about unlikely bad news events. They are prepared to pay comparatively large amounts for lottery tickets where there is a small chance of winning a large price, and at the same time they are willing to buy insurance against large losses that are unlikely to occur. To derive a model that can account for such behavior with a relatively simple preference foundation has been a central topic since at least the work of Friedman and Savage (1963).

Classical expected utility is not able to accommodate optimism and pessimism about small probability events because it must capture all exhibited sensitivity in the utility function. The empirical literature on sensitivity towards probabilities is overwhelming (e.g., Allais 1953, Ellsberg 1962, Kahneman and Tversky 1979, MacCrimmon and Larsson 1979, Cohen and Jaffray 1988, Camerer 1989, Tversky and Kahneman 1992, Wu and Gonzalez 1996, Abdellaoui 2000, Abdellaoui, Vossman and Weber 2005), and a key role for optimism and pessimism has clearly been identified (see Wakker 2001 for a discussion and a thorough review of the descriptive evidence).

For the rank-dependent theories of Quiggin (1981, 1982), Schmeidler (1989) and Tversky and Kahneman (1992), Wakker (2001) has provided a complete and general account of optimism and of pessimism. In those models the role of utility is to account for sensitivity to changes in outcomes and a second instrument, a weighting function (or capacity for choice 
under uncertainty), is measuring the sensitivity towards probabilities (or towards the uncertainty about events). Optimism comes down to a concave weighting function and pessimism is described by a convex weighting function. Wakker (2001, p. 1048-49) particularly highlights the descriptively most plausible pattern for weighting functions, namely the inverse- $S$ shaped ones that are concave for unlikely good news events and convex for unlikely bad news events. A characterization of this important class of probability weighting functions is also provided there.

One difficulty with rank-dependent utility is, unlike with expected utility, that the weighting function complicates the derivation of results in applied work because of the additional mathematics that is required to deal with the weighting functions. For example risk premia and certainty equivalents have a more complex expression. Also, when eliciting utility and its curvature more advanced tools are required (see Wakker and Deneffe 1996, Abdellaoui 2000, Bleichrodt and Pinto 2000). A technical problem with general rank-dependent utility is also that decision weighting may cause unwarranted kinks when the rank of outcomes changes (see Wakker 1994). Expected utility, which is the special case of rank-dependent utility with linear probability weighting, proves to be immune to such jumps and, thus, gains some elegance because of its mathematical simplicity. It appears thus that, when choosing the appropriate model for applications, one must trade off mathematical elegance and simplicity for descriptive realism.

This paper suggests a different approach, namely a compromise as advocated by Bell (1985), Cohen (1992) and recently Chateauneuf, Eichberger and Grant (2007). Instead of adopting general probability weighting functions one can adopt a specific variation that serves both to model the combination of optimism and pessimism with a single weighting function and to maintain mathematical tractability. The specific probability weighting function is linear for non-extreme 
probabilities, but may be discontinuous at 0 and at 1 . As a result, in our model, much of the normative content of expected utility is preserved although substantial descriptive power is incorporated. Wakker (1994) has also highlighted the empirical interest in discontinuities of weighting functions at 0 and at 1 plus continuity on $(0,1)$. The original prospect theory model of Kahneman and Tversky (1979) explicitly used such weighting functions. More recently, Gonzalez and $\mathrm{Wu}$ (1999) tested the linear and discontinuous weighting function, and Kilka and Weber (2001) and Abdellaoui, Vossmann and Weber (2005) provided estimates for the two parameters required to fully describe optimism and pessimism in our model. Parameter estimates for prospect theory specifications were also provided in Abdellaoui, l'Haridon and Zank (2009). They also highlight the role of this class of weighting functions in linking the economic concepts of optimism and pessimism with the interpretation of curvature and elevation originating from psychology.

The existing preference foundations for the type of behavior coined as the non-extreme outcome expected utility, or NEO-EU model by Chateauneuf et al. (2007), have invoked a rich structure on the set of outcomes. We show that those models are also valid for the case that the set of outcomes lacks this additional structure, as is often the case in real applications, e.g., for health states. We adopt the approach of Abdellaoui (2002), which was also followed by Diecidue, Schmidt and Zank (2009) and Zank (2010), and assume a very general set of outcomes. Different to these approaches we will also use a weaker continuity condition and this can complicate our derivation. As will be pointed out in Section 4, and to avoid certain unwarranted cases, some minimal structural assumption on the preference relation is required in order to achieve the familiar uniqueness results for utility and for the weighting function. Thus, we obtain cardinal utility (that is, utility is unique up positive affine transformations) and unique parameters for the weighting function. 
The paper is organized as follows. Section 2 presents general notation. In Section 3 we review expected utility, and a provide specific preference foundation for this classical theory which will help to extend it to NEO-EU preferences. Section 4 highlights the difficulties that can occur when relaxing continuity and the independence axiom underlying expected utility, and presents the structural assumption that we require to derive our main results. Following that, in Section 5 we propose a new preference foundation for NEO-EU. Section 6 concludes. All proofs are deferred to the Appendix.

\section{Preliminaries}

Let $X$ be a set of outcomes. For simplicity of exposition, we assume that $X$ is finite, such that $X=\left\{x_{0}, \ldots, x_{n}\right\}$ for a natural number $n .^{2}$ A lottery is a finite probability distribution over the set $X$. It is represented by $P=\left(p_{0}, x_{0} ; \ldots ; p_{n}, x_{n}\right)$ meaning that probability $p_{j}$ is assigned to outcome $x_{j} \in X$, for $j=0, \ldots, n$. Let $L$ denote the set of all lotteries. The numbers $p_{0}, \ldots, p_{n}$ are nonnegative and sum to 1 . We denote by $\operatorname{supp}(P)=\left\{x_{j} \in X: p_{j}>0\right\}$ the support of $P$.

The set of lotteries $L$ is a mixture space endowed with the operation of probability mixing, i.e., for $P, Q \in L$ and $\alpha \in[0,1]$ the mixture $\alpha P+(1-\alpha) Q$ is also a lottery in $L$. A preference relation, $\succcurlyeq$, is assumed over $L$, and its restriction to subsets of $L$ (e.g., all degenerate lotteries) is also denoted by $\succcurlyeq$. The symbol $\succ$ denotes strict preference, $\sim$ denotes indifference, and $\preccurlyeq$ respectively $\prec$ are the corresponding reversed preferences.

We assume that no two outcomes in $X$ are indifferent, and further, that outcomes are ordered from worst to best, i.e., $x_{0} \prec \cdots \prec x_{n}{ }^{3}$ The strict ranking of outcomes in $X$ is not a

\footnotetext{
${ }^{2}$ None of our results depends on this assumption. The case that $X$ contains only one outcome is trivial. Extensions to the case of infinite $X$ are obtained following a similar approach as in Abdellaoui (2002).

${ }^{3}$ We identify the degenerate lotteries $\left(1, x_{j}\right)$ with the outcome $x_{j}$ for any $j=0, \ldots, n$.
} 
restriction for our theory. This assumption will further simplify the subsequent presentation. It can be shown that, if outcomes in $X$ are allowed to be indifferent, our results also hold using similar arguments as in Abdellaoui (2002) and in Zank (2010).

The aim is to provide preference conditions for $\succcurlyeq$ in order to represent the preference relation over $L$ by a function $V$. That is, $V$ is a mapping from $L$ into the set of real numbers, $I R$, such that for all $P, Q \in L$,

$$
P \succcurlyeq Q \Leftrightarrow V(P) \geq V(Q) \text {. }
$$

This necessarily implies that $\succcurlyeq$ must be a weak order, i.e., $\succcurlyeq$ is complete $(P \succcurlyeq Q$ or $P \preccurlyeq Q$ for all $P, Q \in L)$ and transitive $(P \succcurlyeq Q$ and $Q \succcurlyeq R$ implies $P \succcurlyeq R$ for all $P, Q, R \in L)$.

Expected Utility (EU) holds if the following function represents $\succcurlyeq$ on $L$ :

$$
E U(P)=\sum_{i=0}^{n} p_{i} u\left(x_{i}\right),
$$

where $u$, the utility function, assigns to each outcome a real value. Under EU the utility is cardinal, that is, it is unique up to positive affine transformations.

Let $m_{P}$ denote the worst outcome in $P$ that has positive probability, i.e., $m_{P}=x_{i} \in X$ where $i:=\min \left\{j: p_{j}>0, j=0, \ldots, n\right\}$. Similarly, let $M_{P}$ denote the best outcome in $P$ that has positive probability, i.e., $M_{P}=x_{i} \in X$ where $i:=\max \left\{j: p_{j}>0, j=0, \ldots, n\right\}$. Non-Extreme Outcome Expected Utility (NEO-EU) holds if the preference on $L$ is represented by

$$
N E O(P)=\gamma u\left(m_{P}\right)+(1-\gamma-\delta) E U(P)+\delta u\left(M_{P}\right)
$$

where $0 \leq \gamma, 0 \leq \delta$ and $\gamma+\delta<1$ are uniquely determined constants and utility is like under EU. ${ }^{5}$

\footnotetext{
${ }^{4}$ Essentially, the proofs the apply to indifference sets.

${ }^{5}$ Note that NEO-EU agrees with the special case of rank-dependent utility $R D U(P)=\sum_{i=0}^{n}\left[w\left(p_{i}+\cdots+\right.\right.$ $\left.\left.p_{n}\right)-w\left(p_{i+1}+\cdots+p_{n}\right)\right] u\left(x_{i}\right)$ with $w(0)=0, w(p)=\delta+(1-\gamma-\delta) p$ for $0<p<1$, and $w(1)=1$.
} 
Clearly, if $\gamma=\delta=0$, then NEO-EU coincides with EU. Otherwise, NEO-EU can accommodate more general preferences. Note that NEO-EU "discounts" the expected utility of a lottery by $(1-\gamma-\delta)$ and places the residual weight, separated into proportions $\gamma$ and $\delta$, on the utility of the worst, respectively, on the utility of the best outcome that can obtain in that lottery. A natural interpretation of NEO-EU, therefore, is that this model is "expected utility with the best and worst in mind" (see Chateauneuf, Eichberger and Grant 2007).

An alternative interpretation of the NEO-EU model, one that originates from the analysis of probabilistic attitudes, is also plausible. To illustrate this we recall that probabilistic attitudes are not necessarily globally consistent in the same sense as risk attitudes captured by concave utility under EU. Optimism, for example, is associated with attitudes towards marginal changes in the small probabilities of best outcomes: more attention is given to the small likelihood of obtaining the best outcome in a lottery as compared to the objective probability of that outcome. Pessimism also refers to increased attention above the objective probability of an outcome, however, it refers to the worst outcomes in lotteries (see Wakker 1994, 2001, for comprehensive analyses of probabilistic risk attitudes). The overwhelming empirical evidence suggest that people are ambivalent, and typically exhibit both optimism and pessimism.

We now look at optimism and pessimism as modeled in NEO-EU. We observed before that in the NEO-EU model $(1-\gamma-\delta)$ measures the total deviation from expected utility. Some of this deviation results from pessimistic attitudes and some from optimistic attitudes. As a result, a statement that some agent $\mathrm{A}$ is more affected by these probabilistic risk attitudes than some agent $\mathrm{B}$ means that $\left(1-\gamma_{A}-\delta_{A}\right)>\left(1-\gamma_{B}-\delta_{B}\right)$ (with the subscripts $\mathrm{A}$ and $\mathrm{B}$ indicating the respective agent's NEO-EU parameters) but this does not provide further information about whether agent A is more optimistic (or more pessimistic) than agent B. Suppose now that two lotteries $P$ and $Q$ are evaluated by NEO-EU and that they have the same support. Then, $P$ 
and $Q$ have the same best and the same worst outcome, i.e., $m_{P}=m_{Q}$ and $M_{P}=M_{Q}$ and, as a consequence, the preference between the two lotteries is governed entirely by their expected utility. For example, if $P \succcurlyeq Q$, substitution of NEO-EU gives

$$
\gamma u\left(m_{P}\right)+(1-\gamma-\delta) E U(P)+\delta u\left(M_{P}\right) \geq \gamma u\left(m_{Q}\right)+(1-\gamma-\delta) E U(Q)+\delta u\left(M_{Q}\right),
$$

which, after elimination of common terms is equivalent to $E U(P) \geq E U(Q)$. Hence, NEOEU and EU cannot be distinguished on subsets of lotteries that have the same support (or lotteries with common best and common worst outcomes). For such lotteries, a comparison of risk attitudes of agent $\mathrm{A}$ and agent $\mathrm{B}$ is solely based on the risk attitudes captured by their respective utilities. To extract information about optimistic behavior we need to look at a different set of lotteries.

Consider now the case that the two lotteries $P$ and $Q$ have common worst outcomes but different best outcomes. Then, the weights $\gamma$ and $\delta$ will be influencing preferences. For example, if $P \succcurlyeq Q$, then substitution of NEO-EU and cancellation of common terms gives

$$
E U(P)+\frac{\delta}{(1-\gamma-\delta)} u\left(M_{P}\right) \geq E U(Q)+\frac{\delta}{(1-\gamma-\delta)} u\left(M_{Q}\right)
$$

The weight $\theta:=\delta /(1-\gamma-\delta)$ can be interpreted as a measure of optimism about obtaining the best outcome in the respective lottery relative to the total deviation from expected utility. This measure is consistent in the sense that it is independent of the utility of the best outcome, thus independent of the magnitude of outcomes, but also independent of the positive probability of obtaining the best outcomes.

At first it seems awkward for the measure $\theta$ to be used for interpersonal comparisons of optimism because of the presence of the parameter $\gamma$ in the definition of $\theta$. So, a statement that agent $\mathrm{A}$ is more affected by optimism than agent $\mathrm{B}$ being equivalent to $\theta_{A}>\theta_{B}$ needs some more elaboration. Suppose that agent A and agent B have common utility and common 
parameter $\gamma$. Then $\delta_{A}>\delta_{B} \Leftrightarrow \theta_{A}>\theta_{B}$ under NEO-EU, and the statement that agent A is more optimistic than agent $\mathrm{B}$ is not controversial. However, if, for example, $\delta_{A}=\delta_{B}$ and $\gamma_{A}<\gamma_{B}$ then $\theta_{A}>\theta_{B}$ also holds and the statement that agent $\mathrm{A}$ is more affected by optimism than agent $\mathrm{B}$ is counter-intuitive at a first glance. But note that in this example $\delta_{A}=\delta_{B}$ and $\gamma_{A}<\gamma_{B}$ means that the expected utility of lotteries is discounted more for agent $\mathrm{B}$ than for agent $\mathrm{A}$ and thus agent $\mathrm{A}$ attaches more of the weight taken away from the $\mathrm{EU}$ value of lotteries to best outcomes relative to the weight attached to worst outcomes (i.e., $\theta_{A}>\theta_{B}$ ). So, while optimism and pessimism are two separate components of probabilistic attitudes, in the NEO-EU model both must be understood as being measured relative to the deviation from EU. 6

Using similar arguments one can show that the measure of pessimism about obtaining the worst outcome (relative to the total deviation from EU), $\psi:=\gamma /(1-\gamma-\delta)$, is critical when comparing lotteries with different worst outcomes but common best outcomes. This measure of pessimism is also consistent, similar to the measure of optimism. Hence, an alternative interpretation of NEO-EU as "expected utility with consistent optimism about best outcomes and consistent pessimism about worst outcomes" seems appropriate. As we show in Section 5 below, this consistency requirement is critical for NEO-EU.

Before concluding this section, we recall the two conditions that are satisfied by EU but which may be violated by NEO-EU. The preference relation $\succcurlyeq$ satisfies $v N M$-independence (short for von Neumann-Morgenstern independence) if for all $P, Q, R \in L$ and all $\alpha \in(0,1)$ it holds that

$$
P \succcurlyeq Q \Leftrightarrow \alpha P+(1-\alpha) R \succcurlyeq \alpha Q+(1-\alpha) R
$$

\footnotetext{
${ }^{6}$ This interpretation is analogous to the understanding under expected utility of risk aversion for money as being a deviation from expected value.
} 
That is, the preference between $P$ and $Q$ remains unaffected if both, $P$ and $Q$, are mixed with a common $R$. Note that in the definition of vNM-independence no restrictions apply to the choice of $R$.

The preference relation $\succcurlyeq$ satisfies J-continuity (short for Jensen-continuity) on the set of lotteries $L$ if for all lotteries $P \succ Q$ and $R$ there exist $\rho, \mu \in(0,1)$ such that

$$
\rho P+(1-\rho) R \succ Q \text { and } P \succ \mu R+(1-\mu) Q .
$$

It is well-known (e.g., Herstein and Milnor 1953, Fishburn 1970) that a preference relation $\succcurlyeq$ satisfies weak ordering, Jensen-continuity and vNM-independence on $L$ if and only if it can be represented by expected utility.

As pointed out earlier, if only lotteries with the same support are considered, then EU and NEO-EU are indistinguishable and, therefore, NEO-EU must also satisfy vNM-independence and J-continuity on sets of lotteries with equal support. The difference between the two functions must be in the way that they connect the different sets of lotteries with common support to give an overall representing function for preferences. It will not be sufficient for NEO-EU to restrict vNM-independence and J-continuity to hold only for lotteries with common support. To clarify this, it is worth having another look at the preference foundation for EU and its stability when relaxing some preference conditions. We do this in the following section.

\section{$3 \quad$ Expected Utility}

For any subset of outcomes $Y \subseteq X$ let $L_{Y}$ denote the set of lotteries with support $Y$. For example, $L_{X}$ is the set of lotteries that assign positive probability to each outcome in $X$. Obviously, $L=\cup_{Y \subseteq X} L_{Y}$. We assume throughout this section that $\succcurlyeq$ is a weak order. We note that, if for each $Y \subseteq X$ the preference relation restricted to $L_{Y}$ satisfies J-continuity and 
vNM-independence, then on each set $L_{Y}$ we have (a restriction of) an EU-function representing the preference on that set. Additional information about preferences, which would ensure that these different EU-functions are restrictions of the same EU representation on $L$, may not be available. If we relax only J-continuity to hold on each set $L_{Y}$ but maintain vNM-independence on $L$ there is sufficient information to retain expected utility on $L$. We formally define this restricted property for $\succcurlyeq$, and then we prove that we can still retain EU with the weaker continuity assumption.

The preference relation $\succcurlyeq$ satisfies restricted $J$-continuity if it satisfies J-continuity restricted to the sets $L_{Y}$ for each outcome set $Y \subseteq X$. That is, the following holds for each set $Y \subseteq X$ : for all lotteries $P \succ Q$ and $R$ in $L_{Y}$ there exist $\rho, \mu \in(0,1)$ such that

$$
\rho P+(1-\rho) R \succ Q \text { and } P \succ \mu R+(1-\mu) Q \text {. }
$$

We prove in the Appendix that, in the presence of weak ordering and vNM-independence, restricted J-continuity implies J-continuity on $L$, an thus EU on $L$. Formally, we have the following lemma:

LEMma 1 Assume $\succcurlyeq$ is a weak order on $L$ that satisfies vNM-independence. Then, $\succcurlyeq$ satisfies restricted $J$-continuity if and only if $\succcurlyeq$ satisfies $J$-continuity on $L$.

If we weaken vNM-independence to hold only on lotteries with common support but retain J-continuity on $L$ (and weak ordering) of the preference relation $\succcurlyeq$, then EU still holds. The preference relation $\succcurlyeq$ satisfies restricted $v N M$-independence if the following holds for each set $Y \subseteq X$ : for all lotteries $P, Q$ and $R$ in $L_{Y}$ and any $\alpha \in(0,1)$ we have

$$
P \succcurlyeq Q \Leftrightarrow \alpha P+(1-\alpha) R \succcurlyeq \alpha Q+(1-\alpha) R .
$$

Note that the restricted version of vNM-independence is, on its own, not powerful enough to ensure stochastic dominance on $L$. (First order) stochastic dominance requires for all $P, Q \in L$ 
that $P \succ Q$ whenever $\sum_{i=j}^{n} p_{j} \geq \sum_{i=j}^{n} q_{j}$ for all $j=1, \ldots, n$ and $P \neq Q$. Because, for $j=1, \ldots, n$, the decumulative probabilities $\hat{p}_{j}:=\sum_{i=j}^{n} p_{j}$ of $P$, which indicate the likelihood of receiving outcome $x_{j}$ or better, are at least as high as the corresponding $\hat{q}_{j}$, and for some outcome $x_{k}, 1 \leq k \leq n$, we have $\hat{p}_{k}>\hat{q}_{k}, P$ assigns more probability to better ranked outcomes, and thus it is natural for $P$ to be strictly preferred to $Q$.

The unrestricted version of vNM-independence implies first order stochastic dominance. We formulate this observation as a lemma:

LEMma 2 Assume that $\succcurlyeq$ satisfies vNM-independence on L. Then, $\succcurlyeq$ satisfies stochastic dominance.

Jensen-continuity is weaker than (Euclidean) continuity on $L$, but in the presence of weak order and stochastic dominance it implies the latter (see Abdellaoui 2002, Lemma 18). The preference relation $\succcurlyeq$ satisfies (Euclidean) continuity if for all $P \in L$ the sets $\{Q \in L: Q \succ P\}$ and $\{Q \in L: Q \prec P\}$ are open sets in $L$. As a result it then also follows that restricted vNM-independence is sufficient to derive EU if a weak order satisfies stochastic dominance and J-continuity. We reformulate this remark as a lemma:

Lemma 3 The following two statements are equivalent for a preference relation $\succcurlyeq$ on L:

(i) The preference relation $\succcurlyeq$ on $L$ is represented by expected utility.

(ii) The preference relation $\succcurlyeq$ is a Jensen-continuous weak order that satisfies stochastic dominance and restricted $v N M$-independence.

The utility function $u$ is cardinal.

In terms of probabilities of obtaining an outcome, stochastic dominance implies that an elementary shift of probability $\varepsilon>0$ from a lower ranked outcome $x_{j}$ to an adjacent out- 
come of higher rank $x_{j+1}$ improves a lottery $(j=0, \ldots, n-1)$. Successive elementary shifts can be applied to show that any shift of probability $\varepsilon>0$ from a lower ranked to a higher ranked outcome improves a lottery, which, by repeated applications is equivalent to stochastic dominance.

It has been shown that, when the lotteries are presented in a non-transparent format with multiple elementary shifts being applied, people often violate stochastic dominance (see, e.g., Birnbaum (2008) for a summary of experimental evidence). We could, therefore, have formulated stochastic dominance as a preference for lotteries resulting from improvements by an elementary shift of probability. From an empirical and behavioral point of view, this definition seems more appealing because elementary shifts in probability are transparent and people's choices agree with the preference for the lottery that is improved by an elementary shift.

There is a further implication of vNM-independence that refers to the effect on preference of common elementary shifts in probabilities. We introduce some notation before formally stating this condition, which will also prove useful when considering variations of this principle. Let $P \in L_{Y}$ with $Y=\left\{y_{j}, \ldots, y_{m}\right\}$ for $0 \leq j \leq m \leq n$, and let $z \in X$. For $\varepsilon>0$ let $\left(-\varepsilon, y_{i} ; \varepsilon, z\right) P$ be the lottery in $L_{Y \cup\{z\}}$ in which probability $\varepsilon$ is taken away from an outcome $y_{i} \in Y$ immediately preceding outcome $z$ in the rank and $\varepsilon$ is added to $z$. Formally,

$$
\left(-\varepsilon, y_{i} ; \varepsilon, z\right) P=\left\{\begin{array}{c}
\left(p_{j}, y_{j} ; \ldots ; p_{i}-\varepsilon, y_{i} ; \varepsilon, z ; p_{i+1}, y_{i+1} ; \ldots ; p_{m}, x_{m}\right), \text { if } y_{i} \prec z \prec y_{i+1} \text { and } i<m, \\
\left(p_{j}, y_{j} ; \ldots ; p_{i}-\varepsilon, y_{i} ; \varepsilon+p_{i+1}, y_{i+1} ; \ldots ; p_{m}, x_{m}\right), \text { if } y_{i} \prec z=y_{i+1} \text { and } i<m, \\
\left(p_{j}, y_{j} ; \ldots ; p_{m-1}, y_{m-1} ; p_{m}-\varepsilon, y_{m} ; \varepsilon, z\right), \text { if } y_{m} \prec z .
\end{array}\right.
$$

Because $\left(-\varepsilon, y_{i}, \varepsilon, z\right) P \in L$ it is implicit in this notation that $\varepsilon \leq p_{i}$.

Similarly, for $\varepsilon>0$ let $\left(\varepsilon, z ;-\varepsilon, y_{i}\right) P$ be the lottery in $L_{Y \cup\{z\}}$ in which probability $\varepsilon$ is taken away from an outcome $y_{i} \in Y$ immediately following outcome $z$ in the rank and $\varepsilon$ is added to 
z. Formally,

$$
\left(\varepsilon, z ;-\varepsilon, y_{i}\right) P=\left\{\begin{array}{c}
\left(p_{j}, y_{j} ; \ldots ; p_{i-1}, y_{i-1} ; \varepsilon, z ; p_{i}-\varepsilon, y_{i} ; \ldots ; p_{m}, x_{m}\right), \text { if } y_{i-1} \prec z \prec y_{i} \text { and } i>j, \\
\left(p_{j}, y_{j} ; \ldots ; \varepsilon+p_{i-1}, y_{i-1} ; p_{i}-\varepsilon, y_{i} ; \ldots ; p_{m}, x_{m}\right), \text { if } y_{i-1}=z \prec y_{i} \text { and } i>j, \\
\left(\varepsilon, z ; p_{j}-\varepsilon, y_{j} ; p_{j+1}, y_{j+1} ; \ldots ; p_{m}, y_{m}\right), \text { if } z \prec y_{j} .
\end{array}\right.
$$

Here, too, we require $\left(\varepsilon, z ;-\varepsilon, y_{i}\right) P \in L$, hence, it is implicit in this notation that $\varepsilon \leq p_{i}$.

The preference relation $\succcurlyeq$ satisfies independence of common (elementary) probability shifts on $L$ if

$$
\begin{aligned}
P & \succcurlyeq Q \\
& \Leftrightarrow \\
\left(-\varepsilon, x_{i} ; \varepsilon, x_{i+1}\right) P & \succcurlyeq\left(-\varepsilon, x_{i} ; \varepsilon, x_{i+1}\right) Q,
\end{aligned}
$$

whenever $P, Q,\left(-\varepsilon, x_{i} ; \varepsilon, x_{i+1}\right) P,\left(-\varepsilon, x_{i} ; \varepsilon, x_{i+1}\right) Q \in L$. This property, formulated here in a transparent form that involves only elementary probability shifts, says that joint elementary shifts in probability leave the preference between two lotteries unaffected. The next lemma formally states that vNM-independence implies the weaker independence of common probability shifts.

LEMMA 4 Assume $\succcurlyeq i s$ a weak order on $L$ that satisfies vNM-independence. Then, $\succcurlyeq$ satisfies independence of common probability shifts.

We can now provide an alternative foundation for EU, which will serve as comparison to the NEO-EU foundation that is presented in Section 5. We formulate this result as a theorem:

THEOREM 5 The following two statements are equivalent for a preference relation $\succcurlyeq$ on L:

(i) The preference relation $\succcurlyeq$ on $L$ is represented by expected utility. 
(ii) The preference relation $\succcurlyeq$ is a Jensen-continuous weak order that satisfies stochastic dominance and independence of common probability shifts.

The utility function $u$ is cardinal.

REMARK 6 Theorem 5 remains valid if we replace J-continuity by restricted J-continuity. Theorem 5 also remains valid if one restricts independence of common probability shifts to hold only for lotteries with common support.

Restricting in statement (ii) of Theorem 5 both J-continuity and independence of common probability shifts to hold only for lotteries with common support implies that EU holds on each set $L_{Y}$ for each non-empty set of outcomes $Y \subseteq X$. However, in general, EU need not hold anymore as can be inferred from the fact that NEO-EU also satisfies all those resulting properties. While those properties are necessary for NEO-EU they are not sufficient. The examples in the next section indicate that additional properties must be invoked to derive NEO-EU.

\section{Complications for General Preferences}

In this section we present examples that illustrate difficulties for deriving NEO-EU when considering lotteries over general sets of outcomes. The first example shows that, while for lotteries with common support EU holds (as mentioned in Section 2 this is common to EU and NEO-EU preferences), it need not be the case that NEO-EU holds for a preference on $L$.

EXAmple 7 For a lottery $P$ let $\operatorname{supp}(P)=\left\{y_{0}^{P}, \ldots, y_{k}^{P}\right\}$ for some $0 \leq k \leq n$, and assume that 
$y_{0}^{P} \prec \cdots \prec y_{k}^{P}$. Define the preference relation $\tilde{\succcurlyeq}$ on $L$ as follows:

$$
P \check{\succcurlyeq} Q \Leftrightarrow\left\{\begin{array}{cc}
y_{0}^{P} \succ y_{0}^{Q}, \\
\text { or } y_{i}^{P}=y_{i}^{Q} \text { for all } i<j \text { and } y_{j}^{P} \succ y_{j}^{Q}, & \text { for some } j=1, \ldots, n, \\
\text { or } E U(P) \geq E U(Q), & \text { if } \operatorname{supp}(P)=\operatorname{supp}(Q) \neq X, \\
\text { or } \widetilde{E U}(P) \geq \widetilde{E U}(Q), & \text { if } \operatorname{supp}(P)=\operatorname{supp}(Q)=X,
\end{array}\right.
$$

for some (possibly different) utility functions $u, \tilde{u}: X \rightarrow \mathbb{R}$ that order outcomes according to the ranking $x_{0} \prec \cdots \prec x_{n}$.

The preference in the previous example cannot, in general, be represented by NEO-EU, and the example shows that simply restricting J-continuity and vNM-independence will not retain enough power from the original properties to tie down NEO-EU preferences: one loses, e.g., stochastic dominance. In addition, this example also points at two other important aspects that need to be addressed when the set of outcome is arbitrary. First, for general preferences the existence of common cardinal utility for the representations on sets of lotteries with different support may not be given, and second, a unique pair of parameters $\gamma, \delta$ to measure optimism/pessimism may also not exist. Consider the following example:

EXAmple 8 Let $X=\left\{x_{0}, x_{1}, x_{2}\right\}$ and let $u$ be a utility function defined as $u\left(x_{i}\right)=i / 2$ for $i=0,1,2$, and take $\gamma=\delta=1 / 6$. Let the preference $\succcurlyeq$ on $L$ be represented by

$$
V(P)=1 / 6 u\left(m_{P}\right)+2 / 3 E U(P)+1 / 6 u\left(M_{P}\right) .
$$

Observe that the representation

$$
\widetilde{V}(P)=1 / 3 \tilde{u}\left(m_{P}\right)+1 / 3 \widetilde{E U}(P)+1 / 3 \tilde{u}\left(M_{P}\right),
$$

with $\tilde{u}\left(x_{i}\right)=i\left(=2 u\left(x_{i}\right)\right)$ for $i=0,1,2$ and $\tilde{\gamma}=\tilde{\delta}=1 / 3$ represents the same preference on $L . \square$ 
Example 8 illustrates an identification problem that may occur if there is no possibility to relate the sets of lotteries that have different support. In Example 8 preferences agree with an EUrepresentation on the set of lotteries $A:=L_{\left\{x_{0}, x_{1}, x_{2}\right\}} \cup L_{\left\{x_{0}, x_{2}\right\}} \cup L_{\left\{x_{1}\right\}}$. Accordingly, the ranking of lotteries in $A$ is completely independent of the degrees of optimism or of pessimism. The latter matter only when comparing lotteries from $A$ with lotteries from the sets $L_{\left\{x_{0}\right\}}, L_{\left\{x_{0}, x_{1}\right\}}, L_{\left\{x_{1}, x_{2}\right\}}$, and $L_{\left\{x_{2}\right\}}$. But notice that the image under $V$ of $L=A \cup L_{\left\{x_{0}\right\}} \cup L_{\left\{x_{0}, x_{1}\right\}} \cup L_{\left\{x_{1}, x_{2}\right\}} \cup L_{\left\{x_{2}\right\}}$ is a disconnected set, i.e., it is the union of five disjoint sets. This lack of connectedness means that one can, for example, increase the parameters $\gamma, \delta$ by any arbitrary small $\varepsilon>0$ (here $\varepsilon=1 / 6$ ) and re-scale the (cardinal) utility $u$ appropriately (here $2 u$ is taken) to obtain a different representation, $\widetilde{V}$, that agrees with the ranking of lotteries on $L$ and has different parameters $\tilde{\gamma}, \tilde{\delta}$ that govern pessimism and optimism. Note that for $V$ the measure of optimism is $\delta /(1-\gamma-\delta)=1 / 4$ while for $\widetilde{V}$ the measure of optimism is $\tilde{\delta} /(1-\tilde{\gamma}-\tilde{\delta})=1$.

In Example 8 preferences satisfy weak order, stochastic dominance, restricted J-continuity and independence of common probability shifts restricted to lotteries with common support (by comparison, see statement (ii) of Theorem 5 where the restrictions do not apply). The example shows that, by restricting jointly the independence of common probability shifts and J-continuity to lotteries with common support, structural richness is lost that prevents the simultaneous identification of a unique pair of parameters $\gamma, \delta$ together with cardinal utility. Without uniquely determined measures of optimism or pessimism any interpersonal comparison of these attitudes is impossible. To avoid this extreme situation (like in Examples 7 and 8), we make the following structural assumption for preferences.

Assumption 9 If $n>1$ then, for any $i \in\{1, \ldots, n-1\}$, there exist $P \in L_{\left\{x_{i+1}, \ldots, x_{n}\right\}}$ and $Q \in L_{\left\{x_{i}, \ldots, x_{n}\right\}}$ such that $P \sim Q$, and there exist $R \in L_{\left\{x_{0}, \ldots, x_{i-1}\right\}}$ and $S \in L_{\left\{x_{0}, \ldots, x_{i}\right\}}$ such that 
$R \sim S$

For preferences that satisfy Assumption 9 there is sufficient "overlap" between the images of the sets of lotteries with different support to allow for the identification of cardinal utility. This way, preferences like those in Example 7 are excluded. The principle that will be used for this purpose is a weaker variant of independence of common probability shifts. The preference $\succcurlyeq$ satisfies restricted independence of common (elementary) probability shifts if

$$
P \succcurlyeq Q \Leftrightarrow\left(-\varepsilon, x_{i} ; \varepsilon, x_{i+1}\right) P \succcurlyeq\left(-\varepsilon, x_{i} ; \varepsilon, x_{i+1}\right) Q
$$

whenever $P, Q$ have common best worst and common worst outcomes and $\left(-\varepsilon, x_{i} ; \varepsilon, x_{i+1}\right) P,\left(-\varepsilon, x_{i} ; \varepsilon, x_{i+}\right.$ have common best and common worst outcomes.

Restricted independence of common probability shifts says that the preference between two lotteries with common best and common worst outcomes remains unaffected by a joint elementary probability shift if the resulting lotteries also have (possibly different ) common best and common worst outcomes.

Together with the structural assumption, restricted independence of common probability shifts excludes preferences like those of Example 8 if weak order, restricted J-continuity, stochastic dominance and restricted vNM-independence also hold. A cardinal utility must exist in this case. Yet, as the following example shows, this is still not sufficient to guarantee a unique pair of parameters $\gamma, \delta$, given those properties.

EXAmple 10 Assume that $X=\left\{x_{0}, x_{1}, x_{2}\right\}$ and let $u\left(x_{i}\right)=i$. Define the preference $\succcurlyeq$ through the representation

$$
V(P)=\left\{\begin{array}{cc}
1 / 6 u\left(m_{P}\right)+2 / 3 E U(P)+1 / 6 u\left(M_{P}\right) & \text { if } m_{P}=x_{0} \\
5 / 6 E U(P)+1 / 6 u\left(M_{P}\right), & \text { otherwise. }
\end{array}\right.
$$


Example 10 combines two NEO-EU forms. Specifically, lotteries for which the worst outcome $x_{0}$ can obtain are evaluated by NEO-EU with $\gamma=1 / 6=\delta$, while lotteries where $x_{0}$ is impossible are evaluated by NEO-EU with $\tilde{\gamma}=0, \delta=1 / 6$. This example illustrates that NEO-EU preferences must satisfy additional conditions that pin down the parameter $\gamma$ and the parameter $\delta$ such that these become independent of the magnitude of the worst and best outcomes.

We conclude this section by noting that the preference in Example 8 or Example 10 belong to a larger class called the security and potential level (SPL) preferences (Lopes 1986, Cohen 1992, Diecidue and van de Ven 2008), which have the general representation

$$
S P L(P)=f\left(m_{P}, M_{P}\right) E U(P)+g\left(m_{P}, M_{P}\right)
$$

with a cardinal utility $u$ and some real-valued functions $f\left(m_{P}, M_{P}\right)>0, g\left(m_{P}, M_{P}\right)$ that depend only on the worst and best outcomes within a lottery and such that $S P L$ respects stochastic dominance (see, e.g., the main theorem in Cohen 1992, p.116). Security and potential level preferences agree with NEO-EU preferences if and only if there exist unique $0 \leq \gamma, \delta \leq 1$ with $\gamma+\delta<1$ such that $f\left(m_{P}, M_{P}\right)=(1-\gamma-\delta)$ and $g\left(m_{P}, M_{P}\right)=\gamma u\left(m_{P}\right)+\delta u\left(M_{P}\right)$. In the next section we propose preference conditions that ensure the latter conditions.

\section{Preference Foundation for NEO-EU}

The previous section has illustrated a few complications that may occur if J-continuity and independence of common probability shifts are relaxed. A structural richness assumption for preferences was required to identify cardinal utility. We now focus on the identification of the parameters $\gamma$ and $\delta$ which are uniquely determined under NEO-EU. The fact that these parameters are independent of the magnitude of the worst, respectively, best outcome is characteristic 
for NEO-EU. The following analysis demonstrates an implication of NEO-EU.

Suppose we have found lotteries $P \in L_{\left\{x_{0}, x_{1}, x_{2}\right\}}$ and $Q \in L_{\left\{x_{0}, x_{1}\right\}}$ such that $P \sim Q$. Suppose now that we shift a sufficiently small probability $\varepsilon>0$ from outcome $x_{1}$ to outcome $x_{2}$ and when we do this for both lotteries we obtain two new lotteries ordered as follows ${ }^{7}$

$$
\left(-\varepsilon, x_{1} ; \varepsilon, x_{2}\right) P \preccurlyeq\left(-\varepsilon, x_{1} ; \varepsilon, x_{2}\right) Q
$$

Under weak order, restricted J-continuity, restricted independence of common probability shifts and stochastic dominance we know that EU holds on $L_{\left\{x_{0}, x_{1}, x_{2}\right\}}$. Hence for any $\alpha \in(0,1)$ we have

$$
\alpha\left(-\varepsilon, x_{1} ; \varepsilon, x_{2}\right) P+(1-\alpha)\left[\left(-\varepsilon, x_{1} ; \varepsilon, x_{2}\right) Q\right] \preccurlyeq\left(-\varepsilon, x_{1} ; \varepsilon, x_{2}\right) Q .
$$

The latter preference can also be written as

$$
\left(\alpha(-\varepsilon), x_{1} ; \alpha \varepsilon, x_{2}\right) \alpha P+(1-\alpha)\left[\left(-\varepsilon, x_{1} ; \varepsilon, x_{2}\right) Q\right] \preccurlyeq\left(-\varepsilon, x_{1} ; \varepsilon, x_{2}\right) Q
$$

For $\alpha$ sufficiently small we can find $\theta \geq 0$, such that a additional probability shift from $x_{1}$ now to $x_{2}$ reinstalls indifference:

$$
\left(\alpha(-\varepsilon-\theta), x_{1} ; \alpha(\varepsilon+\theta), x_{2}\right) \alpha P+(1-\alpha)\left[\left(-\varepsilon, x_{1} ; \varepsilon, x_{2}\right) Q\right] \sim\left(-\varepsilon, x_{1} ; \varepsilon, x_{2}\right) Q .
$$

Note that we use $\alpha$ to ensure that the resulting object is a well-defined lottery in $L_{\left\{x_{0}, x_{1}, x_{2}\right\}}$.

Substitution of NEO-EU in the latter indifference implies

$$
\theta\left[u\left(x_{2}\right)-u\left(x_{1}\right)\right]=E U(Q)-E U(P)
$$

and substitution of NEO-EU into $P \sim Q$ gives

$$
\delta\left[u\left(x_{2}\right)-u\left(x_{1}\right)\right]=(1-\gamma-\delta)[E U(Q)-E U(P)] .
$$

\footnotetext{
${ }^{7}$ Note that stochastic dominance excludes a strict preference for the first lottery. Further, EU implies indifference between the lotteries that follow.
} 
From theses two equalities we obtain

$$
\theta=\frac{\delta}{1-\gamma-\delta}
$$

Note that this relationship is independent of outcomes and probabilities. That is, if we, e.g., find $P^{\prime} \in L_{\left\{x_{1}, x_{2}, x_{3}\right\}}$ and $Q^{\prime} \in L_{\left\{x_{1}, x_{2}\right\}}$ such that

$$
P^{\prime} \sim Q^{\prime}
$$

then NEO-EU requires that

$$
\left(\alpha^{\prime}\left(-\varepsilon^{\prime}-\theta\right), x_{2} ; \alpha^{\prime}(\varepsilon+\theta), x_{3}\right) \alpha^{\prime} P^{\prime}+\left(1-\alpha^{\prime}\right)\left[\left(-\varepsilon^{\prime}, x_{2} ; \varepsilon^{\prime}, x_{3}\right) Q^{\prime}\right] \sim\left(-\varepsilon^{\prime}, x_{2} ; \varepsilon^{\prime}, x_{3}\right) Q^{\prime}
$$

for any sufficiently small $\alpha^{\prime}, \varepsilon^{\prime} \in(0,1)$. Hence, the relative measure of optimism $\theta$ must be consistent within and across sets $L_{Y}$.

We can now formulate this property of consistency for $\succcurlyeq$. The preference relation $\succcurlyeq$ satisfies consistent optimism on $L$ if for any $P \in L_{\left\{x_{i}, \ldots, x_{j}\right\}}$ and $Q \in L_{\left\{x_{i^{\prime}}, \ldots, x_{j-1}\right\}}, i \leq i^{\prime}<j, \varepsilon, \alpha \in(0,1)$, and $\theta \geq 0$ with $P \sim Q$ and

$$
\left(\alpha(-\varepsilon-\theta), x_{j-1} ; \alpha(\varepsilon+\theta), x_{j}\right) \alpha P+(1-\alpha)\left[\left(-\varepsilon, x_{j-1} ; \varepsilon, x_{j}\right) Q\right] \sim\left(-\varepsilon, x_{j-1} ; \varepsilon, x_{j}\right) Q
$$

and any $P^{\prime} \in L_{\left\{x_{k}, \ldots, x_{l}\right\}}$ and $Q^{\prime} \in L_{\left\{x_{k^{\prime}}, \ldots, x_{l-1}\right\}}, k \leq k^{\prime}<l$, with $P^{\prime} \sim Q^{\prime}$ it follows that

$$
\left(\alpha^{\prime}\left(-\varepsilon^{\prime}-\theta\right), x_{l-1} ; \alpha^{\prime}\left(\varepsilon^{\prime}+\theta\right), x_{l}\right) \alpha^{\prime} P^{\prime}+\left(1-\alpha^{\prime}\right)\left[\left(-\varepsilon^{\prime}, x_{l-1} ; \varepsilon^{\prime}, x_{l}\right) Q^{\prime}\right] \sim\left(-\varepsilon^{\prime}, x_{l-1} ; \varepsilon^{\prime}, x_{l}\right) Q^{\prime},
$$

for sufficiently small $\varepsilon^{\prime}, \alpha^{\prime} \in(0,1)$ such that $\left(\alpha^{\prime}\left(-\varepsilon^{\prime}-\theta\right), x_{l-1} ; \alpha^{\prime}\left(\varepsilon^{\prime}+\theta\right), x_{l}\right) \alpha^{\prime} P^{\prime}+\left(1-\alpha^{\prime}\right)\left[\left(-\varepsilon^{\prime}, x_{l-1} ; \varepsilon^{\prime}, x_{l}\right)\right.$ $L_{\left\{x_{k}, \ldots, x_{l}\right\}} \cdot$

In a dual manner we formulate consistency for the relative measure of pessimism $\psi$. The preference relation $\succcurlyeq$ satisfies consistent pessimism on $L$ if for any $P \in L_{\left\{x_{i}, \ldots, x_{j}\right\}}$ and $Q \in$ $L_{\left\{x_{i+1}, \ldots, x_{j^{\prime}}\right\}}, i<j^{\prime} \leq j, \varepsilon, \alpha \in(0,1)$, and $\psi \geq 0$ with $P \sim Q$ and

$$
\left(\alpha(\varepsilon+\psi), x_{i} ; \alpha(-\varepsilon-\psi), x_{i-1}\right) \alpha P+(1-\alpha)\left[\left(\varepsilon, x_{i} ;-\varepsilon, x_{i+1}\right) Q\right] \sim\left(\varepsilon, x_{i} ;-\varepsilon, x_{i+1}\right) Q
$$


and any $P^{\prime} \in L_{\left\{x_{k}, \ldots, x_{l}\right\}}$ and $Q^{\prime} \in L_{\left\{x_{k+1}, \ldots, x_{l^{\prime}}\right\}}, k<l^{\prime} \leq l$, with $P^{\prime} \sim Q^{\prime}$ it follows that

$$
\left(\alpha^{\prime}\left(\varepsilon^{\prime}+\psi\right), x_{k} ; \alpha^{\prime}\left(-\varepsilon^{\prime}-\psi\right), x_{k+1}\right) \alpha^{\prime} P^{\prime}+\left(1-\alpha^{\prime}\right)\left[\left(\varepsilon^{\prime}, x_{k} ;-\varepsilon^{\prime}, x_{k+1}\right) Q^{\prime}\right] \sim\left(\varepsilon^{\prime}, x_{k} ;-\varepsilon^{\prime}, x_{k+1}\right) Q^{\prime}
$$

for sufficiently small $\varepsilon^{\prime}, \alpha^{\prime} \in(0,1)$ such that $\left(\alpha^{\prime}\left(\varepsilon^{\prime}+\psi\right), x_{k} ; \alpha^{\prime}\left(-\varepsilon^{\prime}-\psi\right), x_{k+1}\right) \alpha^{\prime} P^{\prime}+(1-$ $\left.\alpha^{\prime}\right)\left[\left(\varepsilon^{\prime}, x_{k} ;-\varepsilon^{\prime}, x_{k+1}\right) Q^{\prime}\right] \in L_{\left\{x_{k}, \ldots, x_{l}\right\}}$.

These two variants of consistency are very weak forms of the probability tradeoff consistency property discussed in Abdellaoui (2002) and Köbberling and Wakker (2003). Related consistency properties appeared in Chateauneuf (1999) and Zank (2010). They are necessary for NEO-EU and in the presence of the preference conditions discussed in the previous section also sufficient. This is our main result and is presented next.

THEOREM 11 The following two statements are equivalent for a preference relation $\succcurlyeq$ on $L$ that satisfies Assumption 9:

(i) The preference relation $\succcurlyeq$ on $L$ is represented by non-extreme outcome expected utility.

(ii) The preference relation $\succcurlyeq$ satisfies (a) weak order; (b) Jensen-continuity restricted to lotteries with common support; (c) first order stochastic dominance; $(d)$ restricted independence of common probability shifts; (e) consistent optimism; and $(f)$ consistent pessimism.

The function $u$ is cardinal, and the parameters $\gamma$ and $\delta$ are uniquely determined (whenever there are more than 2 strictly ordered outcomes).

Note that Theorem 11 supplements the preference foundation for NEO-EU with a tool for measuring the indices of relative optimism $\theta$ and relative pessimism $\psi$ directly from preferences. This makes the result appealing for empirical and experimental analyses. The proof of Theorem 11 is presented in the Appendix. 


\section{Conclusion}

Our main objective in this paper has been to provide a preference foundation for NEO-EU if the set of outcomes is very general. We have shown that, under some minor structural richness of preferences, a foundation of NEO-EU preferences is obtained by relaxing J-continuity and by adjustments of the vNM-independence principle in specific ways. We need to maintain stochastic dominance and relax only the implication of vNM-independence that concerns common probability shifts. The latter are permitted as long as probability is shifted to common outcomes that either were possible in the original pair of lotteries or were impossible. Further, shifts of probabilities are also permitted for best (worst) outcomes but only after appropriate adjustment for optimism (pessimism). Our main theorem shows that the latter implication is the critical difference between EU and NEO-EU. Moreover it demonstrates that NEO-EU has a very rigid means of accommodating optimism and pessimism. The corresponding consistency properties reveal that optimism and pessimism under NEO-EU are completely independent of the magnitude of best or worst outcomes, respectively, but also that optimism and pessimism are independent of the probability of best or worst outcomes. This suggests that the interpretation of the popular NEO-EU model as expected utility with consistent (and constant) optimism and consistent (and constant) pessimism is appropriate.

\section{Appendix: Proofs}

Proof of Lemma 1: Obviously J-continuity on $L$ implies restricted J-continuity. We now prove the reversed implication. There is not much to show if $X$ contains a singleton. For the case that $X=\left\{x_{0}, x_{1}\right\}$ we have to prove that J-continuity holds on $L$, i.e., to demonstrate that 
for any $P \succ Q, P, Q \in L$ and $R \in L$ there exist $\rho, \mu \in(0,1)$ such that

$$
\rho P+(1-\rho) R \succ Q \text { and } P \succ \mu R+(1-\mu) Q .
$$

By definition, any $S \in L$ is a mixture of $x_{0}$ with $x_{1}$ for a unique $\alpha_{S} \in[0,1]$, that is, $S=$ $\alpha_{S} x_{1}+\left(1-\alpha_{S}\right) x_{0}$. From $P \succ Q$ it follows that $\alpha_{P}>\alpha_{Q}$. This follows from vNM-independence and reflexivity of $\succcurlyeq$. Two cases have to be considered: $R \succ Q$ and $R \preccurlyeq Q$.

If $R \succ Q$, then $\alpha_{R}>\alpha_{Q}$ and, thus, for any convex combination $\rho \alpha_{P}+(1-\rho) \alpha_{R}, \rho \in(0,1)$, we have $\rho \alpha_{P}+(1-\rho) \alpha_{R}>\alpha_{Q}$. This implies that $\rho P+(1-\rho) R \succ Q$ for any $\rho \in(0,1)$. Further, vNM-independence implies $\alpha R+(1-\alpha) Q \succ Q$ for all $\alpha \in(0,1)$. Now, take any sufficiently small $\mu \in(0,1)$ such that $\alpha_{P}>\mu \alpha_{R}+(1-\mu) \alpha_{Q}$. Then $P \succ \mu R+(1-\mu) Q$ follows.

If $R \preccurlyeq Q$, then vNM-independence implies $Q \succcurlyeq \alpha R+(1-\alpha) Q$ for all $\alpha \in(0,1)$ and together with $P \succ Q$ and transitivity of $\succcurlyeq$ we obtain $P \succ \mu R+(1-\mu) Q$ for any $\mu \in(0,1)$. Also, because $\alpha_{P}>\alpha_{Q} \geq \alpha_{R}$ there exist $\rho \in(0,1)$ such that $\rho \alpha_{P}+(1-\rho) \alpha_{R}>\alpha_{Q}$, which implies $\rho P+(1-\rho) R \succ Q$

Together the previous two cases imply that J-continuity holds on $L$ if $X$ contains exactly two outcomes.

If $X$ contains more than two outcomes then $X$ also contains a best outcome, $x_{n}$, and a worst outcome, $x_{0}$. Hence, $x_{0} \prec S \prec x_{n}$ holds for any $S \in L$ different from $x_{0}$ or $x_{n}$. This follows from vNM-independence. Thus, weak ordering and vNM-independence together with J-continuity on $L_{\left\{x_{0}, x_{n}\right\}}$ imply that any $S \in L$ different from $x_{0}$ or $x_{n}$ is indifferent to a mixture of $x_{0}$ with $x_{n}$ for some $\alpha_{S} \in(0,1)$. Obviously, by setting $\alpha_{x_{n}}=1$ and $\alpha_{x_{0}}=0$, it follows that for any $S \in L$ there exists a unique $\alpha_{S} \in[0,1]$, with $S \sim \alpha_{S} x_{n}+\left(1-\alpha_{S}\right) x_{0}$, such that $S \succcurlyeq S^{\prime} \Leftrightarrow \alpha_{S} \geq \alpha_{S^{\prime}}$. Now the proof that J-continuity on $L$ holds follows from analogous arguments as in the case that $X$ contains two outcomes. 
This concludes the proof of Lemma 1.

Proof of Lemma 2: Assume that $\succcurlyeq$ satisfies vNM-independence and that for some $P, Q \in L$ we have $\hat{p}_{j} \geq \hat{q}_{j}$ for all $j=1, \ldots, n$ and $P \neq Q$. Suppose that lottery $Q$ equals $P$ except that $\hat{q}_{i}<\hat{p}_{i}$ for some $i \in\{1, \ldots, n\}$. Hence, $P$ is obtained from $Q$ by an elementary shift of probability $p_{i}-q_{i}$ (or equivalently $\hat{p}_{i}-\hat{q}_{i}$ ) from outcome $x_{i-1}$ to outcome $x_{i}$. We prove that $P \succ Q$. The following equivalences follow from repeatedly applying vNM-independence. Define

$$
R:=\left(1-\frac{\hat{p}_{i+1}}{\hat{q}_{i}}, x_{i} ; \frac{p_{i+1}}{\hat{q}_{i}}, x_{i+1} ; \ldots ; \frac{p_{n}}{\hat{q}_{i}}, x_{n}\right)
$$

Then

$$
\begin{aligned}
x_{i} & \succ x_{i-1} \\
& \Leftrightarrow \\
\left(1-\frac{\hat{q}_{i}}{\hat{p}_{i}}\right) x_{i}+\frac{\hat{q}_{i}}{\hat{p}_{i}} R & \succ\left(1-\frac{\hat{q}_{i}}{\hat{p}_{i}}\right) x_{i-1}+\frac{\hat{q}_{i}}{\hat{p}_{i}} R \\
& \Leftrightarrow \\
\left(1-\frac{\hat{p}_{i+1}}{\hat{p}_{i}}, x_{i} ; \frac{p_{i+1}}{\hat{p}_{i}}, x_{i+1} ; \ldots ; \frac{p_{n}}{\hat{p}_{i}}, x_{n}\right) & \succ\left(1-\frac{\hat{q}_{i}}{\hat{p}_{i}}, x_{i-1} ; \frac{q_{i}}{\hat{p}_{i}}, x_{i} ; \frac{p_{i+1}}{\hat{p}_{i}}, x_{i+1} ; \ldots ; \frac{p_{n}}{\hat{p}_{i}}, x_{n}\right) .
\end{aligned}
$$

A subsequent application of vNM-independence by mixing both lotteries in the previous preference with

$$
S:=\left(\frac{p_{0}}{1-\hat{p}_{i}}, x_{0} ; \ldots ; \frac{p_{i-1}}{1-\hat{p}_{i}}, x_{i-1}\right)
$$

by giving probability weight $\left(1-\hat{p}_{i}\right)$ to $S$, gives $P \succ Q$ (using $\left.q_{i-1}=p_{i}-q_{i}+p_{i-1}\right)$.

Suppose now that we have shown that $P^{\prime} \succ Q^{\prime}$ whenever $\hat{p}_{j}^{\prime} \geq \hat{q}_{j}^{\prime}$ for all $j=1, \ldots, n$ and $P^{\prime} \neq Q^{\prime}$ if the set $I:=\left\{j: \hat{p}_{j}^{\prime}>\hat{q}_{j}^{\prime}\right\}$ is of cardinality $|I|<k$ for some $k>1$. We proceed by induction on $|I|$ and show that this statement must be true also for $|I|=k$. Assume that for $P, Q \in L$ we have $\hat{p}_{j} \geq \hat{q}_{j}$ for all $j=1, \ldots, n, P \neq Q$, and $|I|=k$. We prove that $P \succ Q$. Let 
$i$ be the smallest index such that $\hat{p}_{i}>\hat{q}_{i}$ (that is, for $j<i$ we have $\hat{p}_{j}=\hat{q}_{j}$ ). Let now $R, S$ be lotteries defined as follows

$$
\begin{aligned}
R= & \left(1-\frac{\hat{p}_{i+1}}{\hat{q}_{i}}, x_{i} ; \frac{p_{i+1}}{\hat{q}_{i}}, x_{i+1} ; \ldots ; \frac{p_{n}}{\hat{q}_{i}}, x_{n}\right) \\
& \text { and } \\
S= & \left(1-\frac{\hat{q}_{i+1}}{\hat{q}_{i}}, x_{i} ; \frac{q_{i+1}}{\hat{q}_{i}}, x_{i+1} ; \ldots ; \frac{q_{n}}{\hat{q}_{i}}, x_{n}\right) .
\end{aligned}
$$

By the induction assumption it follows that $R \succ S$ because $\hat{p}_{j} / \hat{q}_{i} \geq \hat{q}_{j} / \hat{q}_{i}$ for all $j>i, R \neq S$ and $\left|\left\{j: \hat{p}_{j} / \hat{q}_{i}>\hat{q}_{j} / \hat{q}_{i}\right\}\right|=k-1$. By vNM-independence the following equivalences holds

$$
\begin{aligned}
R & \succ S \\
& \Leftrightarrow \\
\left(1-\frac{\hat{q}_{i}}{\hat{p}_{i}}\right) x_{i}+\frac{\hat{q}_{i}}{\hat{p}_{i}} R & \succ\left(1-\frac{\hat{q}_{i}}{\hat{p}_{i}}\right) x_{i}+\frac{\hat{q}_{i}}{\hat{p}_{i}} S \\
& \Leftrightarrow \\
\left(1-\frac{\hat{p}_{i+1}}{\hat{p}_{i}}, x_{i} ; \frac{p_{i+1}}{\hat{p}_{i}}, x_{i+1} ; \ldots ; \frac{p_{n}}{\hat{p}_{i}}, x_{n}\right) & \succ\left(1-\frac{\hat{q}_{i+1}}{\hat{p}_{i}}, x_{i} ; \frac{q_{i+1}}{\hat{p}_{i}}, x_{i+1} ; \ldots ; \frac{q_{n}}{\hat{p}_{i}}, x_{n}\right) .
\end{aligned}
$$

A further application of vNM-independence by mixing the latter lotteries with

$$
\left(\frac{p_{0}}{1-\hat{p}_{i}}, x_{0} ; \ldots ; \frac{p_{i-1}}{1-\hat{p}_{i}}, x_{i-1}\right)
$$

thereby giving probability weight $\left(1-\hat{p}_{i}\right)$ to the latter lottery, gives $P \succ Q$.

Recall that $P$ and $Q$ were arbitrary with $\hat{p}_{j} \geq \hat{q}_{j}$ for all $j=1, \ldots, n, P \neq Q$, and $|I|=$ $\left|\left\{j: \hat{p}_{j}>\hat{q}_{j}\right\}\right|=k$. Therefore, by induction, it follows that $P \succ Q$ whenever $\hat{p}_{j} \geq \hat{q}_{j}$ for all $j=1, \ldots, n, P \neq Q$. Hence, first order stochastic dominance is derived from vNMindependence, which concludes the proof.

Proof of Lemma 3: That statement (i) implies statement (ii) is immediate. The reversed implication follows from the observation that on $L_{X}$ the properties in statement (ii) imply that 
EU holds on $L_{X}$. Further, as observed in Lemma 18 of Abdellaoui (2002), weak order, stochastic dominance and J-continuity imply the stronger Euclidean continuity on $L$. Therefore, there exists a unique continuous extension of the (previously established) EU-representation on $L_{X}$ to $L$ that represents preferences on $L$. This implies statement (ii). Uniqueness results for utility are carried over from EU on $L_{X}$ to its extension on $L$, thus $u$ is cardinal. This completes the proof of Lemma 3 .

Proof of Lemma 4: Assume that $\succcurlyeq$ satisfies vNM-independence. Take any $P, Q \in L$ such that $P \succcurlyeq Q$ and such that $p_{i}, q_{i}>0$. Suppose, to the contrary, that there exists $\varepsilon>0$ such that for the two lotteries $\left(-\varepsilon, x_{i} ; \varepsilon, x_{i+1}\right) P$ and $\left(-\varepsilon, x_{i} ; \varepsilon, x_{i+1}\right) Q$ the following preference holds

$$
\left(-\varepsilon, x_{i} ; \varepsilon, x_{i+1}\right) P \prec Q_{\varepsilon} .
$$

Then vNM-independence says that

$$
\begin{gathered}
P \succcurlyeq Q \Rightarrow \\
1 / 2 P+1 / 2\left[\left(-\varepsilon, x_{i} ; \varepsilon, x_{i+1}\right) Q\right] \succcurlyeq 1 / 2 Q+1 / 2\left[\left(-\varepsilon, x_{i} ; \varepsilon, x_{i+1}\right) Q\right],
\end{gathered}
$$

and further that

$$
\begin{aligned}
\left(-\varepsilon, x_{i} ; \varepsilon, x_{i+1}\right) P & \prec Q_{\varepsilon} \Rightarrow \\
1 / 2\left[\left(-\varepsilon, x_{i} ; \varepsilon, x_{i+1}\right) P\right]+1 / 2 Q & \prec 1 / 2\left[\left(-\varepsilon, x_{i} ; \varepsilon, x_{i+1}\right) Q\right]+1 / 2 Q .
\end{aligned}
$$

Transitivity the implies that $1 / 2 P+1 / 2\left[\left(-\varepsilon, x_{i} ; \varepsilon, x_{i+1}\right) Q\right] \succ 1 / 2\left[\left(-\varepsilon, x_{i} ; \varepsilon, x_{i+1}\right) P\right]+1 / 2 Q$ which contradicts reflexivity. This shows that $P \succcurlyeq Q \Rightarrow\left(-\varepsilon, x_{i} ; \varepsilon, x_{i+1}\right) P \succcurlyeq\left(-\varepsilon, x_{i} ; \varepsilon, x_{i+1}\right) Q$. The reversed implication is derived in a similar way. Hence, for all (admissible) $\varepsilon>0$ it follows that $P \succcurlyeq Q \Leftrightarrow\left(-\varepsilon, x_{i} ; \varepsilon, x_{i+1}\right) P \succcurlyeq\left(-\varepsilon, x_{i} ; \varepsilon, x_{i+1}\right) Q$. Because $P, Q \in L$ were arbitrary it 
follows that independence of common probability shifts holds. This completes the proof of Lemma 4.

Proof of Theorem 5: That statement (i) implies statement (ii) is immediate. Next we derive the reversed implication and we concentrate on the case that $n>1$ (the other cases are immediate). We write lotteries as decumulative distributions (see also Abdellaoui 2002, Zank 2010 and Diecidue, Schmidt and Zank 2009, who use similar notation). So, instead of $P:=\left(p_{0}, x_{0} ; \ldots ; p_{i}, x_{i} ; p_{i+1}, x_{i+1} ; \ldots ; p_{n}, x_{n}\right)$ we write $P=\left(\hat{p}_{1}, \ldots, \hat{p}_{n}\right)$ with $\hat{p}_{i}=\sum_{j=i}^{n} p_{j}$ for $i=1, \ldots, n$ (for simplicity we drop the outcomes from the notation as well as $\hat{p}_{0}$ which is always equal to 1 ). Then J-continuity, and weak order are defined similarly for decumulative distributions, while stochastic dominance and independence of common probability shifts are translated as monotonicity with respect to decumulative probabilities and additivity, respectively. Formally, monotonicity says that $P \succcurlyeq Q$ whenever $\hat{p}_{i} \geq \hat{q}_{i}$ for all $i=1, \ldots, n$ with $P \succ Q$ if at least one of the inequalities is strict. Additivity says that for all $j=1, \ldots, n$ and $1>\varepsilon>0$

$$
P \succcurlyeq Q \Leftrightarrow\left(\hat{p}_{1}, \ldots \hat{p}_{j-1}, \hat{p}_{j}+\varepsilon, \ldots, \hat{p}_{n}+\varepsilon\right) \succcurlyeq\left(\hat{q}_{1}, \ldots \hat{q}_{j-1}, \hat{q}_{j}+\varepsilon, \ldots, \hat{q}_{n}+\varepsilon\right),
$$

whenever $P, Q,\left(\hat{p}_{1}, \ldots \hat{p}_{j-1}, \hat{p}_{j}+\varepsilon, \ldots, \hat{p}_{n}+\varepsilon\right),\left(\hat{q}_{1}, \ldots \hat{q}_{j-1}, \hat{q}_{j}+\varepsilon, \ldots, \hat{q}_{n}+\varepsilon\right) \in L$.

An important implication of additivity is (comonotonic) independence of common decumulative probabilities or, simply, independence:

$$
\begin{aligned}
\left(\hat{p}_{1}, \ldots \hat{p}_{j-1}, \hat{r}_{j}, \hat{p}_{j+1} \ldots, \hat{p}_{n}\right) & \succcurlyeq\left(\hat{q}_{1}, \ldots \hat{q}_{j-1}, \hat{r}_{j}, \hat{q}_{j+1}, \ldots, \hat{q}_{n}\right) \\
& \Leftrightarrow \\
\left(\hat{p}_{1}, \ldots \hat{p}_{j-1}, \hat{s}_{j}, \hat{p}_{j+1} \ldots, \hat{p}_{n}\right) & \succcurlyeq\left(\hat{q}_{1}, \ldots \hat{q}_{j-1}, \hat{s}_{j}, \hat{q}_{j+1}, \ldots, \hat{q}_{n}\right),
\end{aligned}
$$

whenever $\left(\hat{p}_{1}, \ldots \hat{p}_{j-1}, \hat{r}_{j}, \hat{p}_{j+1} \ldots, \hat{p}_{n}\right),\left(\hat{q}_{1}, \ldots \hat{q}_{j-1}, \hat{r}_{j}, \hat{q}_{j+1}, \ldots, \hat{q}_{n}\right),\left(\hat{p}_{1}, \ldots \hat{p}_{j-1}, \hat{s}_{j}, \hat{p}_{j+1} \ldots, \hat{p}_{n}\right)$, 
$\left(\hat{q}_{1}, \ldots \hat{q}_{j-1}, \hat{s}_{j}, \hat{q}_{j+1}, \ldots, \hat{q}_{n}\right) \in L$.

Independence follows from applying additivity twice, first with $\varepsilon=\left|\hat{r}_{j}-\hat{s}_{j}\right|$ at the $j^{\text {th }}$ decumulative probability and then also at the $(j+1)^{\text {th }}$ decumulative probability.

We know from Abdellaoui (2002) that weak order, J-continuity and monotonicity imply Euclidean continuity and thus, by Debreu (1954), the existence of a representing function $V: L \rightarrow \mathbb{R}$. We use the results of Wakker (1993) to show that by adding (comonotonic) independence the representing function $V$ is additively separable as follows:

$$
V(P)=\sum_{j=1}^{n} V_{j}\left(\hat{p}_{j}\right),
$$

with continuous strictly monotonic functions $V_{1}, \ldots, V_{n}:(0,1) \rightarrow \mathbb{R}$ which are bounded except maybe $V_{1}$ and $V_{n}$ which could be infinite at extreme probabilities (i.e., at 1 , or at 0 , respectively). Further, additivity implies that the functions $V_{j}$ are linear, and hence, proportional to their sum. The $V_{j}$ 's can then be extended continuously to 0 and 1 (this follows from Wakker 1993, Proposition 3.5). Hence, by requiring $V_{j}(0)=0$ for all $j=1, \ldots, n$ we can conclude that there exist positive numbers $a_{j}$ such that

$$
V_{j}(\hat{p})=a_{j} \hat{p} .
$$

We define utility iteratively as $u\left(x_{0}\right)=0$ and $u\left(x_{j}\right)=u\left(x_{j-1}\right)+a_{j}$ for $j=1, \ldots, n$. Therefore, $V_{j}\left(\hat{p}_{j}\right)=\hat{p}_{j} a_{j}=\hat{p}_{j}\left[u\left(x_{j}\right)-u\left(x_{j-1}\right)\right]$ for $j=1, \ldots, n$ with utility $u$ respecting the ranking of the outcomes, i.e., $x_{i} \prec x_{i^{\prime}} \Leftrightarrow u\left(x_{i}\right)<u\left(x_{i^{\prime}}\right)$ for all outcomes $x_{i}, x_{i^{\prime}} \in X$. Hence, statement (i) of the theorem has been derived.

The uniqueness results for utility follow from the fact that the functions $V_{j}$ are jointly cardinal and from the specific construction of $u$.

This completes the proof of Theorem 5 .

Proof OF Remark 6: If we replace J-continuity by restricted J-continuity then Theorem 
5 is true if we also replace $L$ by $L_{Y}$ for any $Y \subseteq X$. We then obtain an expected utility representation $E U_{Y}$ on $L_{Y}$. Independence of common probability shifts (or additivity) implies that for any $Y \subseteq X$ it follows that $u_{Y}=u_{X}$ (up to positive affine transformations). Then statement (i) of Theorem 5 follows for $\succcurlyeq$ on $L$.

If we restrict independence of common probability to lotteries with common support, the proof of Theorem 5 remains valid if we restrict $\succcurlyeq$ to $L_{X}$. Euclidean continuity, which holds on $L$ as a result of J-continuity and stochastic dominance, implies that $E U$ on $L_{X}$ can be extended uniquely to $E U$ on $L$. Statement (i) of Theorem 5 then follows.

This concludes the proof of Remark 6 .

Proof of Theorem 11: First we assume statement (i) and derive statement (ii). We briefly present the arguments: That $\succcurlyeq$ is a weak order follows immediately from NEO-EU. Restricted J-continuity follows from observing that NEO-EU and EU agree on lotteries with common support. Stochastic dominance follows because utility respects the strict ordering of outcomes under NEO-EU. NEO-EU also agrees with EU if lotteries have common best and common worst outcomes, hence, independence restricted to lotteries with common best and common worst outcomes is satisfied. It has been indicated in the main text that NEO-EU satisfies consistent optimism. Similarly, consistent pessimism follows from NEO-EU. Hence, statement (ii) holds.

Next, we assume statement (ii) and derive statement (i). If $X$ contains a single outcome the proof is trivial. For two strictly ranked outcomes weak order and stochastic dominance already ensure that we have a representation of preferences solely based on the probability of obtaining the best outcome. This representation is continuous. Any monotone transformation of this representation (e.g., one which is continuous and linear for probabilities in $(0,1)$ leading 
to NEO-EU, i.e., to statement (i)) is also representing the preference relation. It is well known that in this case uniqueness results are obviously different.

We assume throughout the rest of the proof that $X$ contains at least three strictly ordered outcomes. We note that on $L_{X} \cup\left(\bigcup_{\left\{x_{0}, x_{n}\right\} \subset Y} L_{Y}\right)$, the set of lotteries with $m_{P}=x_{0}$ and $M_{P}=x_{n}$, EU holds with utility function $u$. This follows from observing that the properties in statement (ii) of Theorem 11 imply statement (ii) of Theorem 5 if $\succcurlyeq$ is restricted to $L_{X} \cup$ $\left(\bigcup_{\left\{x_{0}, x_{n}\right\} \subset Y} L_{Y}\right)$. Further, by stochastic dominance and restricted independence of common probability shifts the same $E U$ function also represents $\succcurlyeq$ on any subset of lotteries $L_{Y}$ for $Y \varsubsetneqq X$

We proceed with the proof by induction on $|X|$, the cardinality of $X$.

First we derive NEO-EU for $X=\left\{x_{0}, x_{1}, x_{2}\right\}$. We extend the representing function $E U$ on $L_{X}$ to a NEO-EU representation for $\succcurlyeq$ on $L$. We define utility as $u_{i}:=u\left(x_{i}\right), i=0,1,2$.

Case 1: By Assumption 9 there exists $P \in L_{X}$ and $Q \in L_{\left\{x_{1}, x_{2}\right\}}$ such that $P \sim Q$. It follows that $E U(P) \geq E U(Q)$ by stochastic dominance. Hence there exists a uniquely determined nonnegative number $\rho$ such that $E U(P)+\rho\left[u_{0}-u_{1}\right]=E U(Q)$. We show that $\rho$ is independent of $P, Q$. Suppose that there exists $P^{\prime} \in L_{X}$ and $Q^{\prime} \in L_{\left\{x_{1}, x_{2}\right\}}$ such that $P^{\prime} \sim Q^{\prime}$.

From $P \sim Q$ and $E U(P) \geq E U(Q)$ we know that for any sufficiently small $\varepsilon>0$ we have

$$
\left(\varepsilon, x_{0} ;-\varepsilon, x_{1}\right) P \succcurlyeq\left(\varepsilon, x_{0} ;-\varepsilon, x_{1}\right) Q
$$

As EU holds on $L_{X}$ and $\left(\varepsilon, x_{0} ;-\varepsilon, x_{1}\right) P,\left(\varepsilon, x_{0} ;-\varepsilon, x_{1}\right) Q \in L_{X}$ it follows that for all $\alpha \in[0,1]$ we have

$$
\alpha\left[\left(\varepsilon, x_{0} ;-\varepsilon, x_{1}\right) P\right]+(1-\alpha)\left[\left(\varepsilon, x_{0} ;-\varepsilon, x_{1}\right) Q\right] \succcurlyeq\left(\varepsilon, x_{0} ;-\varepsilon, x_{1}\right) Q .
$$

By restricted J-continuity on $L_{X}$ and stochastic dominance there exists a unique $\psi \geq 0$ such 
that

$$
\left[\alpha(\varepsilon+\psi), x_{0} ;-\alpha(\varepsilon+\psi), x_{1}\right] \alpha P+(1-\alpha)\left[\left(\varepsilon, x_{0} ;-\varepsilon, x_{1}\right) Q\right] \sim\left(\varepsilon, x_{0} ;-\varepsilon, x_{1}\right) Q
$$

for $\alpha$ sufficiently small, such that the resulting object is a lottery in $L_{X}$. Substitution of $E U$ into the latter indifference reveals that $\rho=\psi$. Further, by consistent pessimism,

$$
\begin{aligned}
P & \sim Q, \\
{\left[\alpha(\varepsilon+\psi), x_{0} ;-\alpha(\varepsilon+\psi), x_{1}\right] \alpha P+(1-\alpha)\left[\left(\varepsilon, x_{0} ;-\varepsilon, x_{1}\right) Q\right] } & \sim\left(\varepsilon, x_{0} ;-\varepsilon, x_{1}\right) Q, \\
\text { and } P^{\prime} & \sim Q^{\prime}
\end{aligned}
$$

imply

$$
\left[\alpha^{\prime}\left(\varepsilon^{\prime}+\psi\right), x_{0} ;-\alpha^{\prime}\left(\varepsilon^{\prime}+\psi\right), x_{1}\right] \alpha^{\prime} P^{\prime}+\left(1-\alpha^{\prime}\right)\left[\left(\varepsilon^{\prime}, x_{0} ;-\varepsilon^{\prime}, x_{1}\right) Q^{\prime}\right] \sim\left(\varepsilon^{\prime}, x_{0} ;-\varepsilon^{\prime}, x_{1}\right) Q^{\prime},
$$

for $\varepsilon^{\prime}, \alpha^{\prime} \in(0,1)$ sufficiently small. Substitution of $E U$ into the latter reveals that $E U\left(P^{\prime}\right)+$ $\psi\left[u_{0}-u_{1}\right]=E U\left(Q^{\prime}\right)$. Thus $\rho(=\psi)$ is independent of $P \in L_{X}, Q \in L_{\left\{x_{1}, x_{2}\right\}}$ with $P \sim Q$.

Note that Case 1 also demonstrates that on $L_{X} \cup L_{\left\{x_{0}, x_{2}\right\}} \cup L_{\left\{x_{1}, x_{2}\right\}}$ the functional

$$
\tilde{V}(P):=E U(P)+\psi u\left(m_{P}\right),
$$

with cardinal utility and uniquely determined $\psi$,represents the preference relation $\succcurlyeq$.

Case 2: By Assumption 9 there exists $P \in L_{X}$ and $Q \in L_{\left\{x_{0}, x_{1}\right\}}$ such that $P \sim Q$. It follows that $E U(P) \leq E U(Q)$ by stochastic dominance. Hence there exists a uniquely determined nonnegative number $\sigma$ such that $E U(P)+\sigma\left[u_{2}-u_{1}\right]=E U(Q)$. We show that $\sigma$ is independent of $P, Q$. Suppose that there exists $P^{\prime} \in L_{X}$ and $Q^{\prime} \in L_{\left\{x_{0}, x_{1}\right\}}$ such that $P^{\prime} \sim Q^{\prime}$. From $P \sim Q$ and $E U(P) \leq E U(Q)$ we know that for any sufficiently small $\varepsilon>0$ we have

$$
\left(-\varepsilon, x_{1} ; \varepsilon, x_{2}\right) P \preccurlyeq\left(-\varepsilon, x_{1} ; \varepsilon, x_{2}\right) Q
$$


As EU holds on $L_{X}$ and $\left(-\varepsilon, x_{1} ; \varepsilon, x_{2}\right) P,\left(-\varepsilon, x_{1} ; \varepsilon, x_{2}\right) Q \in L_{X}$ it follows that for all $\alpha \in[0,1]$ we have

$$
\alpha\left[\left(-\varepsilon, x_{1} ; \varepsilon, x_{2}\right) P\right]+(1-\alpha)\left[\left(-\varepsilon, x_{1} ; \varepsilon, x_{2}\right) Q\right] \preccurlyeq\left(-\varepsilon, x_{1} ; \varepsilon, x_{2}\right) Q .
$$

By restricted J-continuity on $L_{X}$ and stochastic dominance there exists a unique $\theta \geq 0$ such that

$$
\left[-\alpha(\varepsilon+\theta), x_{1} ; \alpha(\varepsilon+\theta), x_{2}\right] \alpha P+(1-\alpha)\left[\left(-\varepsilon, x_{1} ; \varepsilon, x_{2}\right) Q\right] \sim\left(-\varepsilon, x_{1} ; \varepsilon, x_{2}\right) Q
$$

for $\alpha$ sufficiently small, such that the resulting object is a lottery in $L_{X}$. Substitution of $E U$ into the latter indifference reveals that $\sigma=\theta$. Further, by consistent optimism,

$$
\begin{aligned}
P & \sim Q, \\
{\left[-\alpha(\varepsilon+\theta), x_{1} ; \alpha(\varepsilon+\theta), x_{2}\right] \alpha P+(1-\alpha)\left[\left(-\varepsilon, x_{1} ; \varepsilon, x_{2}\right) Q\right] } & \sim\left(-\varepsilon, x_{1} ; \varepsilon, x_{2}\right) Q, \\
\text { and } P^{\prime} & \sim Q^{\prime}
\end{aligned}
$$

imply

$$
\left[-\alpha^{\prime}\left(\varepsilon^{\prime}+\theta\right), x_{1} ; \alpha^{\prime}\left(\varepsilon^{\prime}+\theta\right), x_{2}\right] \alpha^{\prime} P^{\prime}+\left(1-\alpha^{\prime}\right)\left[\left(-\varepsilon^{\prime}, x_{1} ; \varepsilon^{\prime}, x_{2}\right) Q^{\prime}\right] \sim\left(-\varepsilon^{\prime}, x_{1} ; \varepsilon^{\prime}, x_{2}\right) Q^{\prime}
$$

for $\varepsilon^{\prime}, \alpha^{\prime} \in(0,1)$ sufficiently small. Substitution of $E U$ into the latter indifference gives $E U\left(P^{\prime}\right)+\theta\left[u_{2}-u_{1}\right]=E U\left(Q^{\prime}\right)$. Thus $\sigma(=\theta)$ is independent of $P \in L_{X}, Q \in L_{\left\{x_{0}, x_{1}\right\}}$ with $P \sim Q$.

Similarly to Case 1, the analysis in Case 2 demonstrates that on $L_{X} \cup L_{\left\{x_{0}, x_{2}\right\}} \cup L_{\left\{x_{0}, x_{1}\right\}}$ the functional

$$
\hat{V}(P):=E U(P)+\theta u\left(M_{P}\right),
$$

with cardinal utility and uniquely determined $\theta$, represents the preference relation $\succcurlyeq$.

Note that $\tilde{V}(P)+\theta u\left(M_{P}\right)$ is also representing the preference $\succcurlyeq$ on $L_{X} \cup L_{\left\{x_{0}, x_{2}\right\}} \cup L_{\left\{x_{1}, x_{2}\right\}}$ (because $M_{P}=x_{2}$ ) and, further, $\psi u\left(m_{P}\right)+\hat{V}(P)$ is also representing the preference $\succcurlyeq$ on 
$L_{X} \cup L_{\left\{x_{0}, x_{2}\right\}} \cup L_{\left\{x_{0}, x_{1}\right\}}$ (because $m_{P}=x_{0}$ ). We therefore conclude that

$$
V(P):=\psi u\left(m_{P}\right)+E U(P)+\theta u\left(M_{P}\right)
$$

is representing the preference $\succcurlyeq$ on $L_{X} \cup L_{\left\{x_{0}, x_{2}\right\}} \cup L_{\left\{x_{1}, x_{2}\right\}} \cup L_{\left\{x_{0}, x_{1}\right\}}$. By stochastic dominance it follows that $V$ can be extended to $x_{0}$ and to $x_{2}$ such that it represents preferences on $L_{X} \cup L_{\left\{x_{0}, x_{2}\right\}} \cup L_{\left\{x_{1}, x_{2}\right\}} \cup L_{\left\{x_{0}, x_{1}\right\}} \cup L_{\left\{x_{0}\right\}} \cup L_{\left\{x_{2}\right\}}$ (which equals $L \backslash L_{\left\{x_{1}\right\}}$ ). It now remains to show that $V$ can be extended to $x_{1}$, so that it represents the preference on all of $L$.

Case 3: By Assumption 9 there exists $P \in L_{X}$ and $Q \in L_{\left\{x_{0}, x_{1}\right\}}$ such that $P \sim Q$, and similarly, there exists $P^{\prime} \in L_{X}$ and $Q^{\prime} \in L_{\left\{x_{1}, x_{2}\right\}}$ such that $P^{\prime} \sim Q^{\prime}$. By stochastic dominance we know that $Q^{\prime} \succ x_{1} \succ Q$, hence, by transitivity, $P^{\prime} \succ x_{1} \succ P$ follows. As $E U$ represents preference on $L_{X}$, is continuous in probabilities, and $L_{X}$ is connected, it follows that there exists a lottery $S \in L_{X}$ such that $S \sim x_{1}$.

Similarly to Case 1 above, we can find sufficiently small $\varepsilon, \alpha \in(0,1)$ and obtain

$$
\left[\alpha(\varepsilon+\psi), x_{0} ;-\alpha(\varepsilon+\psi), x_{1}\right] \alpha P+(1-\alpha)\left[\left(\varepsilon, x_{0} ;-\varepsilon, x_{1}\right) Q\right] \sim\left(\varepsilon, x_{0} ;-\varepsilon, x_{1}\right) Q
$$

from

$$
P \sim Q
$$

By consistent pessimism, the latter two indifferences, together with $S \sim x_{1}$, imply that

$$
\left[\alpha^{\prime}\left(\varepsilon^{\prime}+\psi\right), x_{0} ;-\alpha^{\prime}\left(\varepsilon^{\prime}+\psi\right), x_{1}\right] \alpha^{\prime} S+\left(1-\alpha^{\prime}\right)\left[\left(\varepsilon^{\prime}, x_{0} ; 1-\varepsilon^{\prime}, x_{1}\right)\right] \sim\left(\varepsilon^{\prime}, x_{0} ; 1-\varepsilon^{\prime}, x_{1}\right)
$$

for $\varepsilon^{\prime}, \alpha^{\prime} \in(0,1)$ sufficiently small. Substitution of $V$ into the latter indifference reveals

$$
\psi u\left(m_{S}\right)+E U(S)+\theta u\left(M_{S}\right)=\psi u\left(x_{1}\right)+E U\left(x_{1}\right)+\theta u\left(x_{1}\right) .
$$

It is worth noting that, to derive the latter equation, we could have exploited $P^{\prime} \sim Q^{\prime}$, the analysis in Case 2, and then apply consistent optimism and substitute $V$. We conclude that $V$ represents preference on $L$. 
To complete the proof for the case that $X=\left\{x_{0}, x_{1}, x_{3}\right\}$, we define $\gamma, \delta \in[0,1)$ with $\gamma+\delta<1$ through

$$
\gamma:=\frac{\psi}{1+\theta+\psi} ; \delta:=\frac{\theta}{1+\theta+\psi}
$$

and set $N E O(P):=V(P) /(1+\theta+\psi)$. We observe that

$$
\begin{aligned}
1-\gamma-\delta & =1-\frac{\psi}{1+\theta+\psi}-\frac{\theta}{1+\theta+\psi} \\
& =\frac{1}{1+\theta+\psi},
\end{aligned}
$$

and obtain that

$$
N E O(P)=\gamma u\left(m_{P}\right)+(1-\gamma-\delta) E U(P)+\delta u\left(M_{P}\right),
$$

with cardinal utility $u$ and unique parameters $\gamma, \delta \in[0,1)$ and $\gamma+\delta<1$, represents $\succcurlyeq$ on $L$. This concludes the proof for $|X|=3$.

Assume now that $|X|=r>3$ and that for any non-empty subset $X^{\prime}$ of $X$ the function

$$
N E O_{X^{\prime}}(P)=\gamma_{X^{\prime}} u\left(m_{P}\right)+\left(1-\gamma_{X^{\prime}}-\delta_{X^{\prime}}\right) E U(P)+\delta_{X^{\prime}} u\left(M_{P}\right),
$$

with cardinal utility $u$ and unique parameters $\gamma_{X^{\prime}}, \delta_{X^{\prime}} \in[0,1)$ and $\gamma_{X^{\prime}}+\delta_{X^{\prime}}<1$, represents $\succcurlyeq$ on $\cup_{Y \subseteq X^{\prime}} L_{Y}$.

We need to show that $\gamma_{X^{\prime}}, \delta_{X^{\prime}}$ are independent of $X^{\prime}$ and that functions $N E O_{X^{\prime}}$ are restrictions of a common function $N E O$, that represents the preference relation on $L$.

First, we note that $X_{0}^{\prime}=\left\{x_{0}, \ldots, x_{r-1}\right\}$ and $X_{r}^{\prime}=\left\{x_{1}, \ldots, x_{r}\right\}$ have $\left\{x_{1}, \ldots, x_{r-1}\right\}$ (and any subset of the latter set) in common. Thus, by the induction assumption and the uniqueness results for NEO-representations, it follows that $\gamma_{X^{\prime}}$ and $\delta_{X^{\prime}}$ are independent of $X^{\prime}$. Hence, we can drop the subscripts and use $\gamma$ and $\delta$ henceforth.

It remains to show that $N E O(P)=\gamma u\left(m_{P}\right)+(1-\gamma-\delta) E U(P)+\delta u\left(M_{P}\right)$ does indeed represent $\succcurlyeq$ on $L$. Recall that $N E O$ represents $\succcurlyeq$ on $L_{X} \cup\left(\bigcup_{\left\{x_{0}, x_{n}\right\} \subset Y} L_{Y}\right)$ as it agrees with 
the $E U$ representation on this set.

By Assumption 9 there exists $P \in L_{\left\{x_{1}, \ldots x_{r}\right\}}$ and $Q \in L_{\left\{x_{2}, \ldots x_{r}\right\}}$ such that $P \sim Q$. Because $N E O$ represents the preference $\succcurlyeq$ on $\cup_{Y \subseteq\left\{x_{1}, \ldots x_{r}\right\}} L_{Y}$ it follows that

$$
N E O(P)=N E O(Q)
$$

Division of this equation by $(1-\gamma-\delta)$. Substitution of $\gamma /(1-\gamma-\delta)=: \psi$ and $\delta /(1-\gamma-\delta)=: \theta$ gives

$$
\psi u\left(x_{1}\right)+E U(P)+\theta u\left(x_{r}\right)=\psi u\left(x_{2}\right)+E U(Q)+\theta u\left(x_{r}\right)
$$

or, equivalently,

$$
E U(P)+\psi\left[u\left(x_{1}\right)-u\left(x_{2}\right)\right]=E U(Q) .
$$

From the latter equation we observe that for any sufficiently small $\varepsilon>0$ we obtain

$$
E U\left(\left(\varepsilon, x_{1} ;-\varepsilon, x_{2}\right) P\right)+\psi\left[u\left(x_{1}\right)-u\left(x_{2}\right)\right]=E U\left(\left(\varepsilon, x_{1} ;-\varepsilon, x_{2}\right) Q\right)
$$

and for any $\alpha>0$ such that $\left[\alpha(\varepsilon+\psi), x_{0} ;-\alpha(\varepsilon+\psi), x_{1}\right] \alpha P+(1-\alpha)\left[\left(\varepsilon, x_{0} ;-\varepsilon, x_{1}\right) Q\right] \in L_{\left\{x_{1}, \ldots x_{r}\right\}}$ we obtain

$\alpha E U\left(\left(\varepsilon, x_{1} ;-\varepsilon, x_{2}\right) P\right)+\alpha \psi\left[u\left(x_{1}\right)-u\left(x_{2}\right)\right]+(1-\alpha) E U\left(\left(\varepsilon, x_{1} ;-\varepsilon, x_{2}\right) Q\right)=E U\left(\left(\varepsilon, x_{1} ;-\varepsilon, x_{2}\right) Q\right)$.

This is equivalent to

$$
E U\left(\left[\alpha(\varepsilon+\psi), x_{0} ;-\alpha(\varepsilon+\psi), x_{1}\right] \alpha P+(1-\alpha)\left[\left(\varepsilon, x_{0} ;-\varepsilon, x_{1}\right) Q\right]\right)=E U\left(\left(\varepsilon, x_{1} ;-\varepsilon, x_{2}\right) Q\right)
$$

or, in preference notation,

$$
\left[\alpha(\varepsilon+\psi), x_{0} ;-\alpha(\varepsilon+\psi), x_{1}\right] \alpha P+(1-\alpha)\left[\left(\varepsilon, x_{0} ;-\varepsilon, x_{1}\right) Q\right] \sim\left(\varepsilon, x_{1} ;-\varepsilon, x_{2}\right) Q .
$$

Recall that $P \sim Q$. Further, by Assumption 9 there exists $P^{\prime} \in L_{X}$ and $Q^{\prime} \in L_{\left\{x_{1}, \ldots x_{r}\right\}}$ such that $P^{\prime} \sim Q^{\prime}$. Hence, by consistent pessimism, we obtain

$$
\left[\alpha^{\prime}\left(\varepsilon^{\prime}+\psi\right), x_{0} ;-\alpha^{\prime}\left(\varepsilon^{\prime}+\psi\right), x_{1}\right] \alpha^{\prime} P^{\prime}+\left(1-\alpha^{\prime}\right)\left[\left(\varepsilon^{\prime}, x_{0} ;-\varepsilon^{\prime}, x_{1}\right) Q^{\prime}\right] \sim\left(\varepsilon^{\prime}, x_{1} ;-\varepsilon^{\prime}, x_{2}\right) Q^{\prime}
$$


for $\varepsilon^{\prime}, \alpha^{\prime} \in(0,1)$ sufficiently small. Substitution of $E U$ gives

$$
E U\left(P^{\prime}\right)+\psi\left[u\left(x_{1}\right)-u\left(x_{2}\right)\right]=E U\left(Q^{\prime}\right)
$$

which is equivalent to

$$
N E O\left(P^{\prime}\right)=N E O\left(Q^{\prime}\right)
$$

So, $N E O$ represents preferences on $L_{X} \cup L_{\left\{x_{1}, \ldots x_{r}\right\}}$. This way $\psi$ is uniquely determined.

By a similar argument, starting from $P \in L_{\left\{x_{0}, \ldots x_{r-1}\right\}}$ and $Q \in L_{\left\{x_{0}, \ldots x_{r-2}\right\}}$ with $P \sim Q$ and adding $P^{\prime} \in L_{X}$ and $Q^{\prime} \in L_{\left\{x_{0}, \ldots x_{r-1}\right\}}$ with $P^{\prime} \sim Q^{\prime}$ we obtain, now by exploiting consistent optimism, that $N E O$ represents preferences on $L_{X} \cup L_{\left\{x_{0}, \ldots x_{r-1}\right\}}$. This way $\theta$ is uniquely determined.

It follows that, $\gamma=\psi /(1+\theta+\psi)$ and $\delta=\theta /(1+\theta+\psi)$ are uniquely determined, and that $N E O$ is indeed representing the preference $\succcurlyeq$ on $L$. Hence, by induction, it follows that for any set $X$ consisting of finitely many strictly ranked outcomes the preference $\succcurlyeq$ on $L$ is represented by $N E O$. This proves statement (i).

Uniqueness results for utility and the parameters $\gamma, \delta$ apply by construction. This completes the proof of Theorem 11.

\section{References}

Abdellaoui, Mohammed (2000), "Parameter-free Elicitation of Utility and Probability Weighting Functions," Management Science 46, 1497-1512.

Abdellaoui, Mohammed (2002), "A Genuine Rank-Dependent Generalization of the von NeumannMorgenstern Expected Utility Theorem," Econometrica 70, 717-736. 
Abdellaoui, Mohammed, Olivier l'Haridon and Horst Zank (2009), "Separating Curvature and Elevation: A Parametric Weighting Function," Economics Discussion Papers EDP0901, Economics, University of Manchester, UK, forthcoming in Journal of Risk and Uncertainty.

Abdellaoui, Mohammed, Frank Vossmann and Martin Weber (2005), "Choice-Based Elicitation and Decomposition of Decision Weights for Gains and Losses Under Uncertainty," Management Science 51, 1384-1399.

Allais, Maurice (1953), "Le Comportement de l'Homme Rationnel devant le Risque: Critique des Postulats et Axiomes de l'Ecole Américaine," Econometrica 21, 503-546.

Bell, David E. (1985), "Disappointment in Decision Making under Uncertainty," Operations Research 33, 1-27.

Bleichrodt, Han and Jose Luis Pinto (2000), "A Parameter-Free Elicitation of the Probability weighting Function in Medical Decision Analysis," Management Science 46, 1485-1496.

Birnbaum, Michael H. (2008), "New Paradoxes of Risky Decision Making," Psychological Review 115, 463-501.

Camerer, Colin F. (1989), "An Experimental Test of Several Generalized Utility Theories," Journal of Risk and Uncertainty 2, 61-104.

Chateauneuf, Alain (1999), "Comonotonicity Axioms and Rank-Dependent Expected Utility for Arbitrary Consequences," Journal of Mathematical Economics 32, 21-45.

Chateauneuf, Alain, Jürgen Eichberger and Simon Grant (2007), "Choice under Uncertainty 
with the Best and Worst in Mind: NEO-Additive Capacities," Journal of Economic Theory $137,538-567$.

Chateauneuf, Alain and Peter P. Wakker (1993), "From Local to Global Additive Representation," Journal of Mathematical Economics 22, 523-545.

Cohen, Michèle (1992), "Security Level, Potential Level, Expected Utility: A Three-Criteria Decision Model under Risk," Theory and Decision 33, 101-134.

Diecidue, Enrico, Ulrich Schmidt and Horst Zank (2009), "Parametric Weighting Functions," Journal of Economic Theory 144, 1102-1118.

Diecidue, Enrico and Jeroen van de Ven (2008), "Aspiration level, Probability of Success and Failure, and Expected Utility," International Economic Review 49, 683-700.

Ellsberg, Daniel (1961), "Risk, Ambiguity and the Savage Axioms," Quarterly Journal of Economics 75, 643-669.

Fishburn, Peter C. (1970), Utility Theory for Decision Making. Wiley, New York.

Friedman, Milton and Leonard J. Savage (1948), "The Utility Analysis of Choices Involving Risk," Journal of Political Economy 56, 279-304.

Gonzalez, Richard and George Wu (1999), "On the Shape of the Probability Weighting Function," Cognitive Psychology 38, 129-166.

Herstein, Israel N. and John Milnor (1953), "An Axiomatic Approach to Measurable Utility," Econometrica 21, 291-297.

Kahneman, Daniel and Amos Tversky (1979), "Prospect Theory: An Analysis of Decision under Risk," Econometrica 47, 263-291. 
Kilka, Michael and Martin Weber (2001), "What Determines the Shape of the Probability Weighting Function under Uncertainty?" Management Science 47, 1712-1726.

Köbberling, Veronika and Peter P. Wakker (2003) "Preference Foundations for Nonexpected Utility: A Generalized and Simplified Technique." Mathematics of Operations Research $28,395-423$.

Lopes, Lola L. (1986), "What Naive Decision Makers Can Tell Us about Risk." In Luciano Daboni, Aldo Montesano, \& Marji Lines (eds.), Recent Developments in the Foundations of Utility and Risk Theory, 311-326. Reidel, Dordrecht, The Netherlands.

MacCrimmon, Kenneth R. and Stig Larsson (1979), "Utility Theory: Axioms versus "Paradoxes"." In Maurice Allais and Ole Hagen (Eds), Expected Utility Hypotheses and the Allais Paradox, 333-409, Reidel, Dordrecht, The Netherlands.

Quiggin, John (1981), "Risk Perception and Risk Aversion among Australian Farmers," Australian Journal of Agricultural Economics 25, 160-169.

Quiggin, John (1982), "A Theory of Anticipated Utility," Journal of Economic Behaviour and Organization 3, 323-343.

Schmeidler, David (1989), "Subjective Probability and Expected Utility without Additivity," Econometrica 57, 571-587.

Tversky, Amos and Daniel Kahneman (1992), "Advances in Prospect Theory: Cumulative Representation of Uncertainty," Journal of Risk and Uncertainty 5, 297-323.

von Neumann, John and Oskar Morgenstern (1944, 1947, 1953), "Theory of Games and Economic Behavior." Princeton University Press, Princeton NJ. 
Wakker, Peter P. (1993), “Additive Representations on Rank-Ordered Sets II. The Topological Approach," Journal of Mathematical Economics 22, 1-26.

Wakker, Peter P. (1994) "Separating Marginal Utility and Probabilistic Risk Aversion," Theory and Decision 36, 1-44.

Wakker, Peter P. (2001), "Testing and Characterizing Properties of Nonadditive Measures through Violations of the Sure-Thing Principle," Econometrica 69, 1039-1059.

Wakker, Peter P. and Daniel Deneffe (1996), "Eliciting von Neumann-Morgenstern Utilities when Probabilities Are Distorted or Unknown," Management Science 42, 1131-1150.

Wu, George and Richard Gonzalez (1996), "Curvature of the Probability Weighting Function," Management Science 42, 1676-1690.

Zank, Horst (2010), "Consistent Probability Attitudes," Economic Theory 44, 167-185. 